DOI: $10.1590 / 1089-6891 v 17$ i128347

MEDICINA VETERINÁRIA

\title{
ASPIRAÇÃO FOLICULAR VIDEOLAPAROSCÓPICA COMPARATIVA EM OVELHAS DORPER E SANTA INÊS
}

\section{VIDEOLAPAROSCOPYC OVUM PICK-UP COMPARATIVE IN DORPER AND SANTA INES EWES}

\author{
Endrigo Adonis Braga de Araujo ${ }^{1^{*}}$ \\ Sidnei Nunes de Oliveira ${ }^{1}$ \\ Alexandre de Faria Tabet $^{2}$ \\ Rodrigo Freitas Bittencourt ${ }^{1}$ \\ Marcos Chalhoub ${ }^{1}$ \\ Antonio de Lisboa Ribeiro Filho ${ }^{1}$ \\ ${ }^{1}$ Laboratório de Reprodução Animal, Universidade Federal da Bahia, Salvador, BA, Brasil \\ ${ }^{2}$ Medico Veterinário autônomo, São Paulo, SP, Brasil \\ *Autor para correspondência - adonis.tecvet@yahoo.com.br
}

\begin{abstract}
Resumo
Este estudo teve por objetivo comparar o número de folículos aspirados, oócitos recuperados, qualidade e viabilidade oocitária entre ovelhas das raças Dorper e Santa Inês, submetidas à aspiração folicular por vídeolaparoscopia. Para realização deste estudo, foram efetuadas 118 aspirações foliculares, das quais 65 foram realizadas em ovelhas da raça Dorper e $53 \mathrm{em}$ ovelhas da raça Santa Inês. Os resultados encontrados para as ovelhas Dorper foram 28,31 $\pm 17,42$ folículos/ovelha, 12,21 $\pm 6,74$ oócitos/ovelha, taxa de recuperação de $47,9 \%$ e $8,93 \pm 5,25$ oócitos viáveis/ovelha. Para as ovelhas Santa Inês foram, 22,14 $\pm 16,08$ folículos/ovelha, 12,64 $\pm 8,29$ oócitos/ovelha, taxa de recuperação de $55 \%$ e 8,92 $\pm 6,58$ oócitos viáveis/ovelha. Não houve influência das raças sobre o número de folículos aspirados, oócitos recuperados, taxa de recuperação, qualidade e viabilidade oocitária em aspiração folicular por vídeolaparoscopia.
\end{abstract}

Palavras-chave: folículo; oócito; ovino.

\begin{abstract}
This study aimed to compare the number of aspirated follicles, recovered oocytes, oocyte quality and viability between Dorper and Santa Inês ewes submitted to the videolaparoscopic ovum pick-up. To conduct this study, 118 follicular aspirations were performed, of which 65 were performed in Dorper ewes and 53 in Santa Ines ewes. The results for Dorper sheep were $28.31 \pm 17.42$ follicles/ewe, 12.21 \pm 6.74 oocytes/ewe, recovery rate of $47.9 \%$ and $8.93 \pm 5.25$ viable oocytes/ewe. For Santa Inês ewes the results were $22.14 \pm 16.08$ follicles/ewe, $12.64 \pm 8.29$ oocytes/ewe, recovery rate of $55 \%$ and $8.92 \pm 6.58$ viable oocytes/ewe. There was no influence of breed on the number of aspirated follicles, recovered oocytes, recovery rate, oocyte quality and viability in videolaparoscopic ovum pick-up.

Keywords: follicle; oocyte; sheep
\end{abstract}

Recebido em: 16 fevereiro 2014

Aceito em: 17 novembro 2015 


\section{Introdução}

A expansão na produção de ovinos, observada atualmente, promove uma demanda por biotecnologias da reprodução, com destaque para a produção in vitro de embriões (PIVE), permitindo a exploração intensiva do potencial reprodutivo e genético das fêmeas ${ }^{(1)}$. Entretanto, esta tecnologia ainda não foi devidamente explorada em pequenos ruminantes e diversos fatores podem influenciar em seus resultados.

Dentre os fatores que influenciam o sucesso da PIVE destaca-se a qualidade dos oócitos utilizados na maturação in vitro (MIV) para posterior fecundação, o que torna a colheita oocitária uma etapa fundamental para a execução adequada desta biotecnologia, requerendo uma quantidade suficiente de oócitos de alta qualidade.

Diante disto, a aspiração folicular por vídeolaparoscopia, semelhantemente à aspiração laparoscópica, tem se destacado como importante biotécnica para a recuperação de oócitos em ovinos por ser menos invasiva e traumática quando comparada à recuperação laparotômica ${ }^{(4)}$. Esta técnica apresenta, ainda, a possibilidade de recuperação oocitária em fêmeas com problemas de fertilidade, em períodos de gestação, anestro, senilidade e pré-púberes, possibilitando diminuir o intervalo de gerações e acelerando a propagação de animais de elevado valor genético ${ }^{(5)}$. Além disso, ela pode ser realizada diversas vezes na mesma fêmea sem comprometimento de sua fertilidade ${ }^{(6)}$.

No entanto, alguns fatores relacionados à técnica e inerentes às doadoras necessitam ainda serem avaliados ${ }^{(7)}$, uma vez que não foram encontrados trabalhos que comparem aspirações foliculares entre ovelhas das raças Dorper e Santa Inês.

Desta forma, objetivou-se comparar o número de folículos aspirados, oócitos recuperados, taxa de recuperação, qualidade e viabilidade oocitária entre ovelhas da raça Dorper e Santa Inês submetidas à técnica de aspiração folicular por videolaparoscopia.

\section{Materiais e Métodos}

O estudo foi realizado de acordo com preceitos éticos recomendados pelo Conselho Nacional de Controle de Experimentação Animal (CONCEA), com aprovação do Comitê de Ética no Uso de Animais (CEUA) da Escola de Medicina Veterinária da Universidade Federal da Bahia, sob protocolo $n^{\circ} 30 / 2015$.

O experimento foi realizado na Fazenda Santa Fé, situada no município de Iaçú, região semiárida do Estado da Bahia (longitude oeste $40^{\circ} 26^{\prime}$ e latitude sul $12^{\circ} 33^{\prime}$ ), microrregião da Chapada Diamantina, entre junho de 2010 a junho de 2011.

Para a realização deste estudo, foram efetuadas 118 aspirações foliculares por videolaparoscopia, das quais 65 foram realizadas em 30 ovelhas da raça Dorper e 53 em 30 ovelhas da raça Santa Inês, com intervalo de trinta dias entre aspirações. As ovelhas eram clinicamente hígidas, com três a quatro anos de idade, índice de escore corporal variando de 3 a 4 (escala de 1 a 5), identificadas numericamente por meio da utilização de brincos. Os animais foram mantidos em sistema de criação semi-intensivo com alimentação à base de capim Buffel (Cenchrus ciliaris), suplementado com 250 gramas de ração comercial contendo $18 \%$ de proteína bruta, duas vezes ao dia, além de água e mineralização ad libitum. As ovelhas foram superestimuladas, utilizando-se esponjas intravaginais impregnadas com $60 \mathrm{mg}$ de acetato de Medroxiprogesterona (Progespon ${ }^{\circledR}$, Intervet-Shering Plough, São Paulo, Brasil) em um dia independentemente da fase do ciclo estral (dia 0), permanecendo por um período de dez dias. No oitavo dia do protocolo (dia 8) aplicou-se 37,5 $\mu \mathrm{g}$ de D-cloprostenol (Prolise ${ }^{\circledR}$, Tecnopec, São Paulo, Brasil), 300UI de eCG (Novormon ${ }^{\circledR}$, Intervet-Shering Plough, São Paulo, Brasil) e 200UI de FSHp (Foltropin ${ }^{\circledR}$, Tecnopec, São Paulo, Brasil) via intramuscular. Procederam-se às aspirações foliculares por videolaparoscopia no décimo dia do protocolo (Figura 1). 


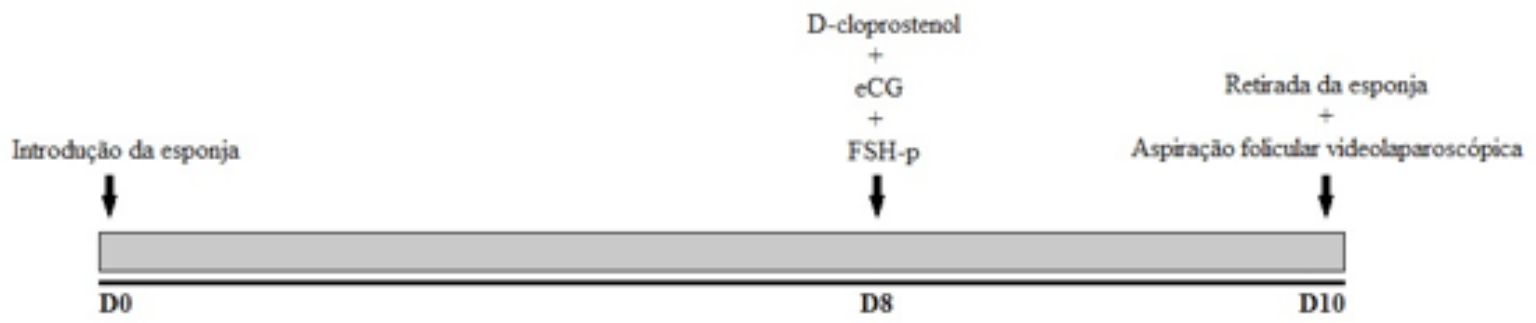

Figura 1: Protocolo de superestimulação ovariana para realização de aspiração folicular.

Após o protocolo de superestimulação, jejum alimentar de 24 horas e hídrico de 12 horas, as doadoras foram sedadas com Xilazina 0,2 mg $/ \mathrm{kg}$ (Rompun ${ }^{\circledR}$, Bayer, São Paulo - SP, Brasil) por via intramuscular. Após 10 minutos, foi fixado um cateter número 18 na veia jugular das ovelhas que foram colocadas na maca cirúrgica em decúbito dorsal. Na sequência, foi feita a tricotomia e antissepsia da região ventral do abdome. A indução anestésica foi realizada com Diazepam 0,35mg/kg (Hipolabor, Belo Horizonte - MG, Brasil) por via intravenosa associada a Ketamina (Dopalen ${ }^{\circledR}$, Vetbrands, Paulínia - São Paulo, Brasil) diluída em soro fisiológico em infusão contínua na proporção de 1:100 $(0,01)$, que também foi utilizada na manutenção anestésica.

Com a maca inclinada em 45 graus, estando a pelve elevada, efetuaram-se três incisões de pele, sendo a primeira localizada a aproximadamente $5,0 \mathrm{~cm}$ lateral à linha média no lado direito, próximo à glândula mamária, para introdução do primeiro trocarte $(5 \mathrm{~mm})$ para passagem da óptica $(5 \mathrm{~mm}, 30$ graus). Por meio desse trocarte, o abdome foi insuflado com ar ambiente proveniente da bomba da fonte de luz.

Uma segunda incisão foi realizada no lado oposto à primeira para introdução de outro trocarte $(5$ $\mathrm{mm}$ ) para passagem da pinça de apreensão ovariana. A última incisão foi feita cranial à primeira para introdução de trocarte $(5 \mathrm{~mm})$ para penetração da bainha acoplada à mangueira e agulha de aspiração. A mangueira foi acoplada em um tubo coletor contendo PBS (Gibco BRL, Life Technologies BV, Breda, The Netherlands) a $37^{\circ} \mathrm{C}$, acrescido de heparina (Liquemine ${ }^{\circledR}$, Roche, Rio Janeiro, Brasil), e este conectado a uma bomba de vácuo. Todos os folículos observados foram puncionados e contados, independentemente do seu tamanho ou grau de maturação. Ao final, ambos os ovários foram banhados com solução fisiológica e as dermorrafias realizadas com pontos tipo Wolf, com fio nylon 2-0.

O conteúdo folicular aspirado foi colocado em placas de Petri, observado ao esteriomicroscópio, a fim de se identificar e contar os oócitos recuperados. Estes foram classificados qualitativamente conforme os critérios morfológicos adotados por Rahman et al. ${ }^{(8)}$, sendo subdivididos em cinco grupos: G1- oócitos grau I (excelente); G2- oócitos grau II (bom); G3- oócitos grau III (regular); Desn. - Desnudo; e Cit. Irr. - citoplasma irregular. Apenas aqueles com pelo menos uma camada de células do cumulus e eventuais granulações citoplasmáticas foram considerados viáveis (G1-G3).

As variáveis estudadas - número de folículos, número de oócitos, taxa de recuperação (número de oócitos/número de folículos e o resultado multiplicado por 100) e viabilidade - foram comparadas entre os grupos experimentais por meio do PROC GLM, utilizando-se o teste T de Student, e a variável qualidade oocitária que não apresentou distribuição normal foi comparada por meio do teste de Mann-Whitney. O nível de significância adotado foi de 5\%. Os dados deste experimento foram analisados utilizando-se o pacote estatístico SAS, versão seis (1996).

\section{Resultados e Discussão}

Comparando-se o número de folículos, número de oócitos, taxa de recuperação e viabilidade oocitária 
entre as raças Dorper e Santa Inês, não foi verificada diferença estatística $(\mathrm{P}>0,05)$ os resultados encontrados estão demonstrados na Tabela 1.

Tabela 1: Média e desvio padrão do número de folículos, número de oócitos, taxa de recuperação e viabilidade oocitária de ovelhas Dorper e Santa Inês

\begin{tabular}{ccccc}
\hline Raças & $\begin{array}{c}\text { Folículos } \\
\mathbf{N}\end{array}$ & $\begin{array}{c}\text { Oócitos } \\
\mathbf{N}\end{array}$ & $\begin{array}{c}\text { Taxa de } \\
\text { recuperação } \\
\mathbf{( \% )}\end{array}$ & Viabilidade oocitária \\
\hline Dorper & $28,31 \pm 17,42$ & $12,21 \pm 6,74$ & $47,9 \pm 22,44$ & $8,93 \pm 5,25(64,14 \%)$ \\
Santa Inês & $22,14 \pm 16,08$ & $12,64 \pm 8,29$ & $55 \pm 30,84$ & $8,92 \pm 6,58(71,43 \%)$ \\
\hline
\end{tabular}

O protocolo de superestimulação utilizado neste experimento mostrou-se eficiente. Teixeira et al. ${ }^{(6)}$, por outro lado, obtiveram resultado inferior $(11,3 \pm 3,0$ folículos/ovelha Santa Inês) ao encontrado neste experimento ao efetuarem estimulação ovariana com única aplicação de FSH e eCG 12h após detecção do estro, que foi sincronizado utilizando-se esponja intravaginal impregnada com Medroxiprogesterona por seis dias e aplicação de D-Cloprostenol e eCG no quinto dia do protocolo. Do mesmo modo, Baldassarre et al. (9), utilizando doadoras caprinas das raças Saanen e Toggenburg e protocolo de única aplicação de FSH + eCG, obtiveram resultados inferiores $(17,9 \pm 8,0)$ aos obtidos nesta pesquisa. Mesmo utilizando protocolo "one shot", as pequenas variações efetuadas por Teixeira et al. ${ }^{(6)}$ podem ter influenciado no resultado; já em relação ao resultado encontrado por Baldassarre et al. ${ }^{(9)}$, pode se tratar de uma característica da espécie trabalhada.

Gibbons et al. ${ }^{(10)}$ buscaram maximizar a colheita de oócitos para produção in vitro de embriões em pequenos ruminantes, e encontraram média de 14,4 folículos por ovelha da raça Merino, após estimulação do desenvolvimento folicular com dose única de FSH associado a eCG efetuada $48 \mathrm{~h}$ após inserção de esponja intravaginal impregnada de Medroxiprogesterona, repetida de quatro em quatro dias num total de quatro tratamentos. Assim como nos trabalhos supracitados, a média de folículos por doadoras obtida por esses autores foi inferior às médias foliculares encontradas em Dorper e Santa Inês nesta pesquisa, podendo-se inferir que há influência da espécie e raça no número de folículos após estimulação ovariana, uma vez que, mesmo com pequenas variações, os autores citados utilizaram aplicação única de FSH e eCG.

Pierson et al. ${ }^{(11)}$, estimulando o desenvolvimento folicular com uma única dose de FSH associado a eCG 36 horas antes de remover a esponja de Medroxiprogesterona e a realização da aspiração folicular guiada por laparoscopia, obtiveram, durante o programa, um total de 6996 folículos aspirados, perfazendo uma média de 14,3 $\pm 5,3$ folículos por doadora, em desacordo aos resultados deste experimento. No entanto, Koeman et al. ${ }^{(12)}$ compararam a resposta folicular em cabras prépúberes e adultas, em um trabalho cujo objetivo foi analisar a capacidade de desenvolvimento de oócitos cultivados em meio semi-definido após a recuperação laparoscópica. Estes autores utilizaram o mesmo protocolo de estimulação folicular relatado até o momento e obtiveram 18,4 $\pm 1,1$ folículos por doadoras adultas e 32,0 $\pm 3,7$ por doadoras pré-púberes, resultado superior aos obtidos neste trabalho, mostrando a possível influência da idade na atividade ovariana.

Os 28,3 folículos por ovelhas Dorper e 22,1 folículos por ovelhas Santa Inês encontrados nesta pesquisa foram superiores aos resultados de Rodriguez et al. ${ }^{(13)}$ com 17,4 folículos, Cox \& Alfaro ${ }^{(14)}$ com 17,2 folículos, Wieczoreck et al. ${ }^{(3)}$ com 4,6 folículos e Avelar et al. ${ }^{(15)}$ com 14,8 folículos em média. Isso se deve, provavelmente, ao fato de terem utilizado diferentes protocolos de superestimulação, variando de várias aplicações de FSH a uma única aplicação de eCG, que podem ter influenciado diretamente na resposta ovariana.

No presente estudo, também foi demonstrada a eficiência da técnica de aspiração folicular videolaparoscópica, obtendo-se bom resultado em relação ao número de oócitos por doadora e, por consequência, uma boa taxa de recuperação; no entanto, mais investigação é necessária para se alcançar maior eficiência. 
Em trabalhos consultados ${ }^{(3,5,6,12,13,16)}$, nos quais realizou-se laparotomia, laparoscopia e vídeolaparoscopia para aspiração folicular, observou-se variação de 3,1 a 25 oócitos/doadora e taxa de recuperação oscilando de $24,7 \%$ a $90,7 \%$, demonstrando a influência da técnica e dos equipamentos nas variáveis estudadas.

Teixeira et al. ${ }^{(6)}$, utilizando a mesma técnica de aspiração folicular, obtiveram resultado inferior em número de oócitos $(5,8 \pm 2,3)$ e taxa de recuperação semelhante $(51,7 \%)$ e Alberio et al. ${ }^{(17)}$ relataram resultados inferiores tanto em número de oócitos $(4,9 \pm 1,3)$ quanto na taxa de recuperação $(33,6 \%)$. De forma semelhante, os resultados desse experimento divergem dos relatados por Wieczorek et al. ${ }^{(3)}$ (3,1 oócitos/doadora), Rodriguez et al. ${ }^{(13)}$ (8,7 oócitos/doadora), Gibbons et al. ${ }^{(10)}$ (6,8 oócitos/doadora) e Avelar et al. ${ }^{(15)}$ (11,1 oócitos/doadora), que realizaram aspirações foliculares por laparoscopia. No entanto, as taxas de recuperação dos autores citados foram semelhantes ou superiores aos resultados deste experimento, sendo elas $66,8 \%, 49,9 \%, 47,0 \%$ e $74,7 \%$, respectivamente.

$\mathrm{Na}$ literatura ${ }^{(5,6,13)}$, observa-se uma gama de variações nos equipamentos e calibragens predominando a falta de clareza nas metodologias. Os calibres das agulhas variaram de 16 a $20 \mathrm{G}$ e, em relação à pressão de aspiração e fluxo, nota-se uma falta de padrão que dificulta a execução de comparações. Há autores que descrevem o fluxo em $\mathrm{mL} \mathrm{H}_{2} \mathrm{O}$ /minuto e outros em gotas/minuto, que provavelmente são medidas cedidas pelo equipamento utilizado, além de a pressão de aspiração nem sempre estar exposta na metodologia.

Confrontando-se o número de oócitos obtidos por doadoras neste trabalho e os encontrados por Baldassare \& Karatzas ${ }^{(4)}$ em caprinos (13,4 oócitos/doadora) e Basso et al. ${ }^{(1)}$ em aspirações comerciais em ovinos (14,3 oócitos/doadora), observou-se uma similaridade entre os resultados.

$\mathrm{Na}$ comparação da qualidade oocitária entre os grupos experimentais não foi observada diferença $(\mathrm{p}>0,05)$. Os resultados estão apresentados na Figura 2.

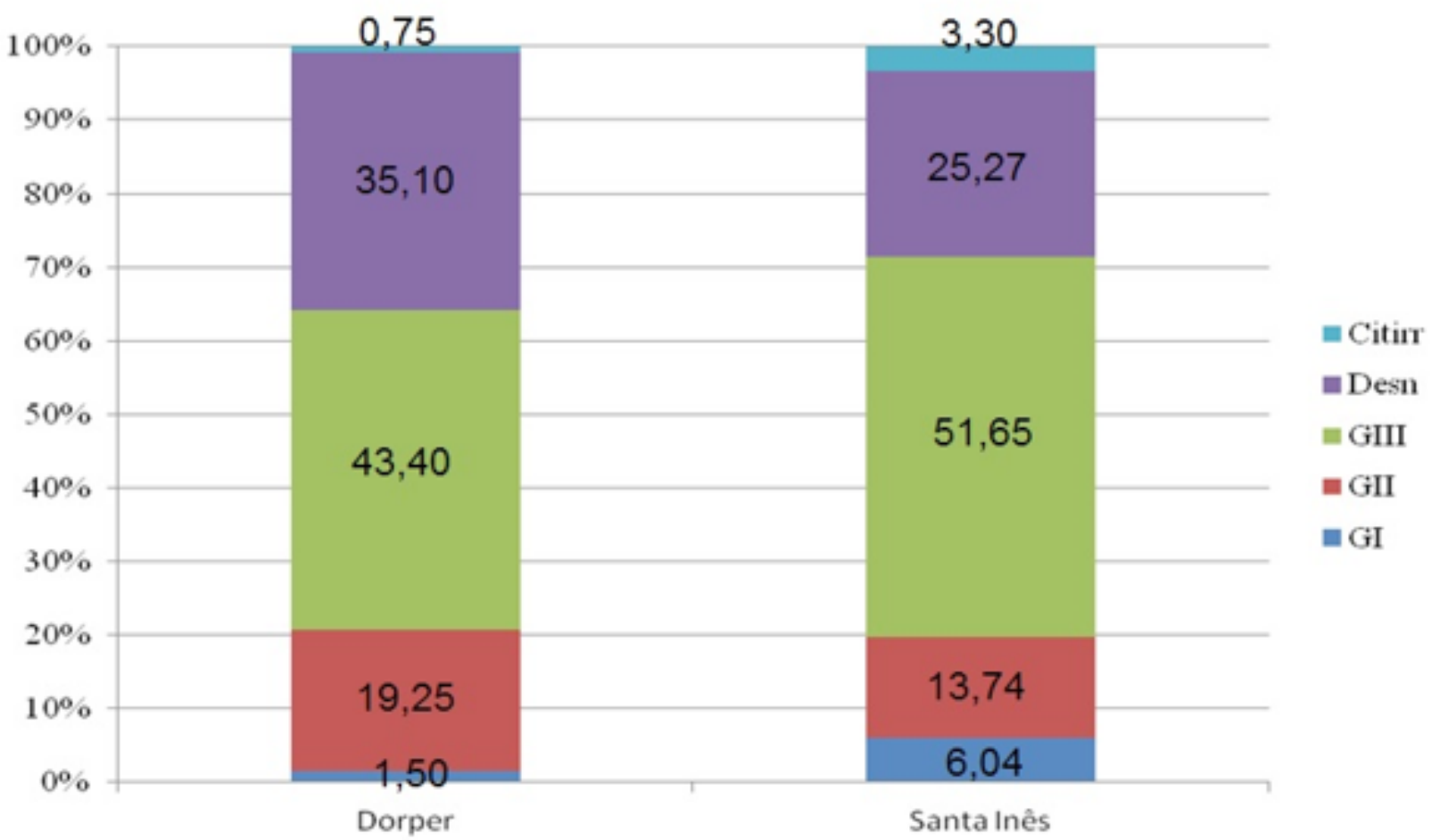

Figura 2: Porcentagem acumulada da qualidade oocitária de ovelhas das raças Dorper e Santa Inês. GI - oócitos grau I (excelente), GII - oócitos grau II (bom), GIII - oócitos grau III (regular), Desn - oócitos desnudos e Citirr. - oócitos com citoplasma irregular. 
Max et al. ${ }^{(18)}$ realizaram aspirações por laparotomia em 5 doadoras ovinas Santa Inês, após superestimulação com FSH+eCG 36h antes das aspirações, das quais obtiveram 49 oócitos, classificados da seguinte forma: 46,9\% Grau I; 16,3\% Grau II; 18,4\% Grau III; 10,2\% Desnudos; e $8,2 \%$ citoplasma irregular. Abdullah et al. ${ }^{(16)}$ realizaram aspirações foliculares por laparoscopia em 15 cabras, após estimulação folicular utilizando FSH+hCG 36h antes das aspirações, e obtiveram $29,7 \% \pm 8,3$ oócitos grau I, $22,1 \% \pm 6,7$ oócitos grau II, $41,4 \% \pm 9,6$ oócitos grau III e 7,5\% $\pm 3,6$ oócitos desnudos.

Em comparação à qualidade oocitária resultante desta pesquisa, notou-se diferença entre a distribuição da qualidade, sendo maior a proporção de Grau III e Desnudos, o que caracteriza a obtenção de oócitos de qualidade inferior quando comparado aos trabalhos supracitados. Esse resultado pode ter sido influenciado pelos diversos fatores relacionados à técnica e aos equipamentos utilizados, dentre os quais, de grande relevância, o diâmetro da agulha e o fluxo e a pressão de aspiração, que influenciam diretamente a qualidade oocitária ${ }^{(13)}$.

A viabilidade oocitária, objetivo final da aspiração folicular como passo inicial para o desenvolvimento de biotecnologias como a produção in vitro de embriões, foi satisfatória, alcançando-se resultados superiores e semelhante aos resultados obtidos por Rodriguez et al. ${ }^{(13)}(58,1 \%)$ e Gibbons et al. ${ }^{(10)}$ $(63,2 \%)$; entretanto, inferior aos resultados de Max et al. ${ }^{(18)}(81,6 \%)$, Alberio et al. ${ }^{(17)}(87,0 \%)$ e Koeman et al. ${ }^{(12)}$, em dois grupos estudados, fêmeas adultas $(82,0 \%)$ e pré-púberes $(87,0 \%)$.

Oócitos considerados viáveis são aqueles classificados como Grau I, II e III; por isso, uma vez que a qualidade oocitária for baixa, refletirá diretamente na viabilidade. Todos os fatores relacionados à técnica e a calibragem dos aparelhos utilizados para aspiração folicular que interferem na qualidade são responsáveis também pela quantidade de oócitos viáveis ${ }^{(19,20)}$.

\section{Conclusão}

Não há influência entre ovelhas da raça Dorper e Santa Inês quanto ao número de folículos aspirados, oócitos recuperados, taxa de recuperação, qualidade e viabilidade oocitária quando submetidas à aspiração folicular por vídeolaporoscopia.

\section{Referências}

1. Basso AC, Martins JFP, Ferreira CR, et al. Biotecnologia da Reprodução na Espécie Ovina: Produção in vitro de Embriões Ovinos: Aspectos da Técnica de Aspiração Folicular e do Tratamento Hormonal de Doadoras. O Embrião. 2008;38(10):8-11.

2. Wani NA. In vitro maturation and in vitro fertilization of sheep oocytes. Small Ruminant Research. 2002;44(2):89-95.

3. Wieczorek J, Kosenyuk Y, Cegła M, et al. A new concept in 83 laparoscopic ovum pick-up (OPU) in sheep - efficiency of method and morphology of 84 recovered oocytes. Reproduction in Domestic Animals. 2010;10(1):39-48.

4. Baldassarre $\mathrm{H}$, Karatzas $\mathrm{CN}$. Advances assisted reproduction technologies (ART) in goats. Animal Reproduction Science.2004;82:255-266.

5. Baldassarre H, Wang B, Kafidi N, et al. Advances in the production and propagation of transgenic goats using laparoscopic ovum pick-up and in vitro embryo production reproduction technologies. Theriogenology. 2002;57:275-284.

6. Teixeira PPM, Padilha LC, Oliveira MEF, et al. Laparoscopic ovum collection in sheep: Gross and microscopic evaluation of the ovary and influence on ooctye production. Animal Reproduction Science. 2011;127:169-175.

7. Cognié Y, Poulin N, Locatelli Y, et al. State-of-the-art production, conservation and transfer of in vitro 
produced embryos in small ruminants. Reproduction, Fertility and Development. 2004;16:437-445.

8. Rahman ANMA, Abdullah RB, Wan Khadijah WE. Goat embryo development from in vitro matured oocytes of heterogeneous quality through intracytoplasmic sperm injection technique. Biotechnology. 2007;6:373-382.

9. Baldassarre H, Rao KM, Neveu N, et al. Laparoscopic ovum pickup followed by in vitro embryo production for the reproductive rescue of aged goats of high genetic value. Reproduction, Fertility and Development. 2007;19:612-616.

10. Gibbons A, Pereyra Bonnet F, Cueto MI, et al. Procedure for maximizing oocyte harvest for in vitro embryo production in small ruminants. Reproduction in Domestic Animals. 2007;42:423-426.

11. Pierson J, Wang B, Neveu N, et al. Effects of repetition, interval between treatments and season on the results from laparoscopic ovum pick-up in goats. Reproduction ${ }_{2}$ Fertility and Development. 2004;16:795-799.

12. Koeman J, Keefer CL, Baldassarre H, et al. Developmental competence of prepubertal and adult goat oocytes cultured in semi-defined media following laparoscopic recovery. Theriogenology. 2003;60:879-889.

13. Rodríguez C, Anel L, Alvarez M, et al. Ovum Pick-up in Sheep: a Comparison between Different Aspiration Devices for Optimal Oocyte Retrieval. Reproduction in Domestic Animals. 2006;41:106-113.

14. Cox JF, Alfaro V. In vitro fertilization and development of OPU derived goat and sheep oocytes. Reproduction of Domestic Animals. 2007;42:83-87.

15. Avelar SRG, Moura RR, Sousa FC, et al. Oocyte production and in vitro maturation in Canindé goats following hormonal ovarian stimulation. Animal Reproduction Science. 2012; 9(1):27-32.

16. Abdullah RB, Liow SL, Rahman ANMA, Chan WK, Wan-Khadijan WE, Ng SC. Prolonging the interval from ovarian hyperstimulation to laparoscopic ovum pick-up improves oocyte yield, quality, and developmental competence in goats. Theriogenology. 2008;70:765-771.

17. Alberio R, Olivera, J, roche A, et al. Performance of a modified ovum pick-up system using three different FSH stimulation protocols in ewes. Small Ruminant Research. 2002;46:81-87.

18. Max MC, Santos GMG, Melo-Sterza FA, et al. In vitro embryo production in sheep: Pregnancy after long periods of oocyte and embryo transport. Small Ruminant Research. 2012;105:286-289.

19. Gibbons A, Bonnet FP, Cueto MI, et al. Colheita de oócitos guiada por laparoscopia em caprinos e ovinos. Acta Scientiae Veterinariae. 2008;36(2):223-230.

20. Crocomo LF, Marques Filho WC, Landim-Alvarenga FC, et al. Peculiaridades da coleta de oócitos para produção in vitro de embriões ovinos. Rev. Bras. Reprod. Anim. 2012;36(1):25-31. 